Y. MATSUMOTO, J. SAWAMURA, Y. MURATA, T. NISHIKATA*, R. YAZAKI*, T. OHSHIMA* (YAMAGUCHI UNIVERSITY, UBE, AND KYUSHU UNIVERSITY, FUKUOKA, JAPAN)

Amino Acid Schiff Base Bearing Benzophenone Imine as a Platform for Highly Congested Unnatural $\alpha$-Amino Acid Synthesis J. Am. Chem. Soc. 2020, 142, 8498-8505.

\section{Copper-Catalyzed Synthesis of Nonnatural $\alpha$-Amino Acids}<smiles>[2H]C([3H])(Br)C(N=C(c1ccccc1)c1ccccc1)C(=O)O</smiles>

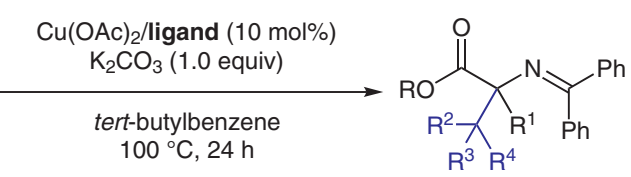

(2.0 or 4.0 equiv)

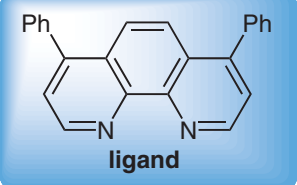

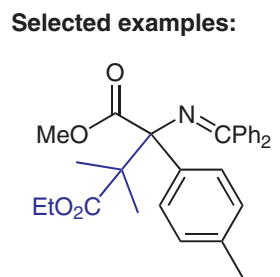

$72 \%$ yield<smiles>COC(=O)C(N=Cc1ccccc1)(C(=O)OC)C1(C(=O)O)CCCCC1</smiles>

$73 \%$ yield<smiles>CCOC(=O)C(C)(C)[C@](N=Cc1ccccc1)(C(=O)OCC)C(=O)OC1CC(C)CC[C@@H]1C(C)(C)[NH3+]</smiles>

$66 \%$ yield, $\mathrm{dr}=93: 7^{\mathrm{a}}$

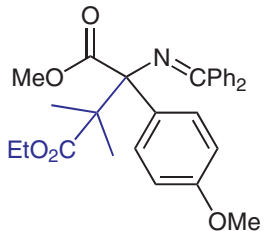

$85 \%$ yield<smiles>COC(=O)C(N=Cc1ccccc1)(C(=O)OC)C1(C(=O)O)CCCC1</smiles>

$78 \%$ yield<smiles>CCOC(=O)C(C)(C)C(N=CP)(C(=O)OC)c1ccc(Cl)cc1</smiles>

$93 \%$ yield<smiles>COC(=O)C1(C(N=Cc2ccccc2)(C(=O)OC)c2ccccc2)CCC1</smiles>

$52 \%$ yield

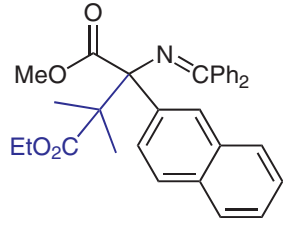

$86 \%$ yield

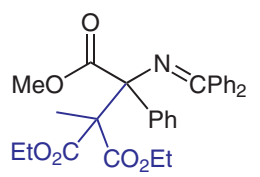

$37 \%$ yield

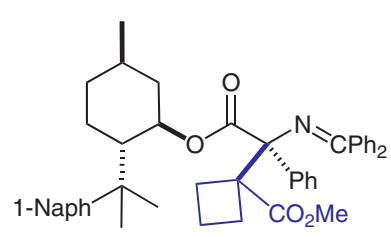

$23 \%$ yield, $d r=96: 4^{a}$

${ }^{\mathrm{a} C}$ Conditions: $\mathrm{Cu}(\mathrm{OAc})_{2}(10 \mathrm{~mol} \%)$, ligand (10 mol\%), KOt-Bu (4.0 equiv), THF, r.t., 6 h.

Significance: Nonnatural amino acids are invaluable building blocks in synthetic organic chemistry. The authors have developed a copper-catalyzed approach for the synthesis of nonnatural $\alpha$-amino acids.
Comment: This copper-catalyzed $\alpha$-alkylation reaction provides the desired $\alpha$-tetrasubstituted $\alpha$-amino acids in moderate to high yields. The approach can be applied to a diastereoselective synthesis of $\alpha$-tetrasubstituted $\alpha$-amino acids by using a chiral auxiliary. 